\title{
Verify Your Runs
}

Klaus Havelund and Allen Goldberg

Kestrel Technology, Palo Alto, California, USA

\{havelund,goldberg\}@esestreltechnology.com

http://www.kestreltechnology.com

\section{Introduction}

A program verifier determines whether a program satisfies a specification. Ideally verification is achieved by static analysis without executing the code. However, program verification is unsolvable in general. The interactive approach, for example with a human guiding a theorem prover, does not in practice scale to large software systems. Some restricted kinds of specifications can, however, be checked automatically, for example type definitions. Also static analysis of properties such as un-initialized variables, null-pointer de-referencing, and arraybound violations scales to production programs on the order of hundreds of thousands of lines of code. Even concurrency-related problems such as data races and deadlocks can to some extent be checked statically, although often resulting in false positives. However, going beyond these simple properties to arbitrarily complex behavior specification and scaling to ever-growing production program size is undoubtedly a challenge, and in our opinion we cannot expect regular economic use of program verification of arbitrary properties to be fully achieved within the 15 year time horizon of the challenge.

Hence, we will probably have to accept that parts of the verification task will remain as proof obligations. It is reasonable to not throw such proof obligations away, but to monitor them during program testing, or in the operations phase. In the latter case, one can program reactions to property violations to achieve some form of fault protection. We call the scientific discipline that studies the monitoring of properties during program execution runtime verification 11. Much work has been done in this area within recent years.

In this paper we shall outline and classify some current approaches to runtime verification and describe our contributions. We shall describe how we intend to further contribute to this work in the framework of the Grand Verification Challenge. The paper does not address the topic of test case generation although runtime verification is a part of this subject. That is, an effectful test case generation framework needs to support the generation of test cases, where a test case consists of inputs to the program together with an oracle that will inspect the output of the program (including inspection of its internal behavior) when executed on that input. Generation of the oracle is the runtime verification part. We believe that runtime verification is a rich subject on its own. 


\section{Specification-Based Runtime Verification}

Specification-based runtime verification consists of monitoring a program's execution against a user-provided specification of intended program behavior. The many approaches to program specification logics have lead to differing styles of runtime verification. One can consider a spectrum of monitoring approaches, ranging from monitoring of predicate assertions stating properties about a single state at a single program location, to monitoring of temporal assertions stating properties about temporally separated states at multiple program locations identified by automated program instrumentation. We shall discuss the techniques along four dimensions:

- Location quantification: whether the logic allows to quantify over locations in the program to be monitored. Monitors evaluate when certain program locations are reached during program execution. If the monitoring code is executing in-line, these locations will contain the monitoring code itself. If monitoring is off-line, the locations will contain event generators, that will send events to the monitors that run in some more or less loose form of synchronization with the code. Locations can either be specified individually, by identifying each of them explicitly, or they can be quantified over, as in Aspect Oriented Programming, covering many locations with one declaration, for example "before every call of any method defined in class $C$, evaluate monitor $M$ ".

- Temporal quantification: whether the logic allows quantification over time points. For example, whether one can express properties of the form: "whenever a call of method close occurs then in the past there has been a call of the open method". Some temporal logics only allow to state ordering relationships, while others go further and allow to state relative or absolute time values.

- Data quantification: whether the logic allows binding and referral (forward or backward in time) to values across states. For example, "whenever a call of method close $(f)$ occurs, with a file argument $f$, then in the past there has been a call of open $(f)$ of the same file $f . "$ Obviously, data quantification presumes temporal quantification.

- Abstract data specification: whether abstract states mapping variable names to values can be defined together with an abstraction that relates concrete program states to abstract specification states.

In the following we shall classify a collection of monitoring approaches along these dimensions.

Assertions. Runtime checks are assertions inserted at specific locations in the code. Assertions were introduced in Java 1.4 such that the programmer can write assertions of the form assert $\psi$ for a Java predicate $\psi$, at explicit program locations. Assertions do not directly support location, temporal or data quantification, nor abstract data specification. 
Pre-Post Conditions. Pre-post conditions is an extension of the assert statement, where the programmer explicitly indicates where checks should be performed, namely before and after method calls, hence not supporting location quantification. However, a post condition typically relates the value of variables at the start and the end of the method. Thus this is a restricted form of temporal and data quantification. The Eiffel language 25] has long embodied this idea, and recently so has JML 41, the Java Modeling Language. In Eiffel there is no provision for abstract data specification, while in JML there is. The Larch Shared Language approach 44] supports abstract (axiomatic) data specification in combination with pre-post conditions.

Invariants. Invariants, as found in for example Eiffel and JML, express properties about a single state, and are required to hold at all locations where data consistency can be expected, specifically at the completion of method calls, and at the limit after every variable update. Hence this is an example of a logic supporting location quantification. Since an invariant asserts a property of just the current state it does not support temporal or data quantification.

State Machine Notations and Process Algebras. In state machines/automata and process algebras, the specification is an abstract program, and runtime verification dynamically checks that the executing program is a refinement of the abstract program. That is, the instrumented program locations correspond to abstract program states, and monitoring checks the required state sequencing and that each concrete program state satisfies (via an abstraction function) the properties of a corresponding abstract state. This form of specification supports temporal quantification. The Jass system [40] monitors a combination of JML and CSP process algebra. Alternating automata, supporting AND as well as OR states, have shown to be particularly convenient for monitoring logics as demonstrated in [22 28 27]. Also state charts 32] offer this combination of AND and OR states. An example illustrating the use of state machines for monitoring is the TLChart system 24, that monitors a combination of temporal logic and state machines. An extension of simple state machines are timed automata, where time constraints can be put on states (one can only be in a state for a certain time period) and on transitions. One such system for runtime verification of timed automata is T-UPPAAL [56] and another similar system is described in 13. T-UPPAAL also generates test cases. All the systems mentioned above monitor finite traces against finite trace automata. In 20] is described a technique for monitoring against Omega automata: automata that normally accept infinite traces. This is specifically useful for monitoring automata generated from specifications originally targeted for model checkers such as SPIN [54]. In [30] is described an algorithm for synthesizing finite trace monitoring algorithms from LTL specifications, inspired by similar algorithms used for synthesizing infinite trace Omega automata from LTL specifications.

Temporal Assertions. While automata and process algebras are operational in nature, temporal logics are declarative. Temporal logics have operators that relate arbitrary states, and hence support full temporal quantification, and can 
in many cases allow more succinct specifications. Pre-post conditions support a simple form of temporal quantification by relating two states (the pre-state and the post-state). In the commercial Temporal Rover system [22, one writes past time and future time temporal logic formulas at specific program locations, that get evaluated whenever that program location is reached. This tool hence supports temporal quantification but not location quantification. The MaC system [43. supports temporal past time assertions and location quantification by allowing instrumentation of method calls and variable updates. MaC also allows abstract data specifications referenced as the propositions of the temporal logic. An interesting logic is the future time temporal logic PSL [46] adopted by the hardware industry. In metric temporal logics one can state properties about time. Several such systems have been developed, for example 2257. Regular expressions, and extended regular expressions allowing negation, appear to be very useful for writing certain properties that in temporal logic would become more complicated to state. Such a system is described in [51. A generalization of metric logics are data logics supporting data quantification, where one can reason about data values existing at different time points. Such systems are described in $2327 / 21$. Temporal logics are often mapped to automata, although other interpretations are possible, such as for example described in 4936, where rewriting is used to interpret temporal logic for monitoring.

General Purpose Specification Languages. A monitoring language may be a complete formal specification language, in the style of ASML [7, Maude [17, PVS 47, VDM 60, RAISE 48, or Specware 53. This is the approach taken at Microsoft where ASML (Abstract State Machine Language) 7] is used for runtime verification as part of a general test case generation framework. Clearly such an approach supports abstract data specification. These full specification languages usually have executable subsets which resemble a programming language, be it functional or state-based. This observation can be exploited by having the specification language be an extension of the programming language, an approach taken in Spec\# 9], Microsoft's extension of the work in 7.

\section{Predictive Runtime Verification}

As with testing, the effectiveness of runtime verification depends on the choice of test suite. For concurrent systems this becomes even more serious because this is compounded by the many possible execution paths of a non-deterministic program. This raises the question of whether there are properties that can be checked on one or a small number of execution traces and still identify bugs with high probability (if such exist). The answer is affirmative due to recent work on what we call predictive runtime verification.

In predictive runtime verification a property $P$ to be monitored is replaced with a stronger property $Q$, i.e. for all inputs $x, Q(x) \rightarrow P(x)$. Furthermore if $\forall x P(x)$ then $Q(x)$ for most $x$ (few false positives) but if $\exists x \neg P(x)$ then $\neg Q(x)$ for most $x$ (good detection). It turns out that for certain problems finding such $Q$ is possible. 
One of the earliest successes was the Eraser algorithm [50] for detecting data races, that was implemented in Compaq's Visual Threads tool 33. This algorithm checks a single execution trace in order to determine whether there are any potentials for data races: the situation where two threads access a shared variable simultaneously. This work has later been extended to cover other forms of data races, such as higher level data races [5] and atomicity violations 6 2963. Also deadlocks of the dining philosopher format can be checked in this manner [15. A generalized predictive analysis framework is presented in [52]. In most of the above mentioned systems, the properties are programmed directly as algorithms in a traditional programming language. Attempts have, however, been made to express the properties in logic [10. These are often data oriented properties that are best expressed in a monitoring logic appropriate for expressing data quantification and location quantification.

Concurrent target systems may be modified by inclusion of wait statements or modifications to schedulers, so that a fuller range of non-deterministic behaviors are exhibited during testing. Such modifications can be combined with predictive analysis. This is discussed in the overview paper [26] and in [14].

\section{Instrumentation}

Instrumentation is the modification of the target system with additional code that informs the monitor of events and data values relevant to the monitored properties, such as the taking of a lock, the entry into a method, or the update of a variable. This can be achieved through source code instrumentation, for example using Aspect Oriented Programming (AOP) as supported by AspectJ [8]; through byte-code instrumentation, BCEL [12] being an example byte-code instrumentation tool; or through object code instrumentation, with Valgrind 58 being an example. The Java-MOP system described in 16 is a generalized framework for instrumenting Java programs specifically for runtime verification. Instrumentation can, however, also be done through debugging interfaces, modification of the runtime system or virtual machine, or through operating system or middleware services. In our work we have used byte-code instrumentation and aspect oriented programming to instrument code.

It is worth noting that runtime verification techniques are starting to appear within the aspect oriented programming community. In a traditional AOP language such as AspectJ [42, an aspect specifies augmentations/modifications to a program, that may add functionality or specify a correctness property and appropriate actions to be executed when the property is violated. Traditionally actions are weaved into the program at program points, specified by so called pointcuts. A recent trend is to augment the pointcut language to include predicates on the execution trace. Solutions have been offered for augmenting AOP with regular expressions 2, future time linear temporal logic [5519], state machines [59, and grammars 62.

Naive instrumentation can cause significant degradation of performance and is a significant concern for most systems, especially real time systems. Static 
analysis can be used as a technique for optimizing runtime monitoring. This is a dual but equivalent view to that presented in the introduction, namely that runtime monitoring is used to verify residual properties that remain unverified by (static) program verification.

\section{Our Previous Work}

In this section we briefly outline our own and close colleague's work in runtime verification. Some of our early work 34 was done in predictive runtime verification of concurrent Java programs and resulted in a tool for performing predictive deadlock and Eraser-like data race analysis on Java programs, guiding the Java PathFinder (JPF) model checker 61] to confirm the warnings discovered by the much faster predicative analysis. Instrumentation was done by modifying the Java Virtual Machine of JPF. The work on predictive runtime verification was later reimplemented and elaborated in the Java PathExplorer (JPaX) tool [37 35]. Specifically the deadlock analysis algorithm was improved to yield fewer false warnings 15. In [14] is described an approach where such deadlock warnings are fed into a testing framework, where detected potential deadlock cycles are used to control the execution of the program in an attempt to confirm the deadlocks. Other recent results on predictive runtime verification includes work that goes beyond low-level data races on single variables, and includes detection of high-level data races on collections of variables [5], and detection of out-dated copies of shared variables [6]. JPaX also supports specification-based runtime verification. The Maude rewriting system [17] is used to define new logics [4936. This has proved extremely elegant since Maude is well suited for defining the syntax and semantics of a logic. In [38.39] we describe how to synthesize very efficient algorithms based on dynamic programming for monitoring past time logic.

In more recent work we decided to develop a runtime verification framework for Java in Java. Eagle [1] is a powerful temporal kernel language supporting temporal quantification and capable of modeling all of the temporal logics and most of the specification paradigms mentioned in this overview. Eagle is an extension of propositional logic with three temporal kernel operators, recursion, and parameterization over formulas in the logic as well as over data values. Formula parameterization allows the user to define new temporal combinators, and hence new temporal logics. The language therefore directly supports the definition of new specification patterns of the kind illustrated in [45. Data parameterization allows to define properties relating data values from different points in time, hence supporting data quantification. Due to these constructs Eagle can define various forms of past and future time linear temporal logics, real-time logics, interval logics, extended regular expressions and state machines. Eagle furthermore supports abstract data specification in that formulas are interpreted on an abstract state defined as a Java class, and referred to as the Eagle state. In principle there is a stratification of the propositional language and the logic proper so that Java may be replaced by a high-level specification language. The user must define an abstraction mapping from concrete program 
states to abstract Eagle states. At each instrumentation location in the monitored target system, a method representing the abstraction function is called to update the Eagle state. Noting that Eagle supports the definition of state machines, we see that Eagle hence supports both data refinement and control refinement. A recent extension of Eagle supports automated program instrumentation [19, hence location quantification, using the aspect oriented programming tool AspectJ [8]. In previous work we developed the jSpy tool [31], which instruments Java byte-code. A jSpy instrumentation specification consists of a set of rules, each of which consists of a condition on byte-code and an instrumentation action stating what to report when byte-codes satisfying the condition are executed. The reported events are then picked up by the monitors that in turn check for various user provided properties. Eagle has been used within a test-case framework as described in 34 .

\section{Future Work}

As a scientific discipline specification-based runtime verification does not face the same difficult problems as, say, model checking or theorem proving, and is likely closer to become part of practical software development environments. However, the discipline faces unsolved problems concerned with choice of specification notations, monitoring algorithms, code instrumentation, as well as social issues such as the usual resistance amongst software developers to write formal specifications in addition to the code itself. We feel that predictive runtime verification should be part of any development system since it is very effective, fully automated, requires no specifications, and essentially imposes only minor cost to the programmer. The challenge is to identify other problems that lend themselves to this form of analysis. Concerning specification-based runtime verification, choosing the right specification formalism is critical to the success of the approach. The formalism must be simple, yet powerful, and/or, it could be an already accepted notation, such as UML. We will continue experimenting with Eagle, but we will also investigate other formalisms in order to achieve the optimal balance between simplicity, efficiency and effectiveness. Amongst work not mentioned is that on generating specifications from runs [18. We intend to extend our work in this direction.

\section{References}

1. 1st - 5th Workshops on Runtime Verification (RV 2001 - RV 2005). ENTCS, vol. 55(2), 70(4), 89(2), 113. Elsevier Science Direct. Amsterdam (to be published, 2001-2005), http://www.runtime-verification.org

2. Allan, C., Avgustinov, P., Kuzins, S., de Moor, O., Sereni, D., Sittamplan, G., Tibble, J., Christensen, A.S., Hendren, L., Lhoták, O.: Adding Trace Matching with Free Variables to AspectJ. In: OOPSLA 2005 (2005)

3. Artho, C., Barringer, H., Goldberg, A., Havelund, K., Khurshid, S., Lowry, M., Pasareanu, C., Rosu, G., Sen, K., Visser, W., Washington, R.: Combining TestCase Generation and Runtime Verification. Theoretical Computer Science 336(23), 209-234 (2005), Extended version of [4] 
4. Artho, C., Drusinsky, D., Goldberg, A., Havelund, K., Lowry, M., Pasareanu, C., Roşu, G., Visser, W.: Experiments with Test Case Generation and Runtime Analysis. In: Börger, E., Gargantini, A., Riccobene, E. (eds.) ASM 2003. LNCS, vol. 2589, pp. 87-107. Springer, Heidelberg (2003)

5. Artho, C., Havelund, K., Biere, A.: High-Level Data Races. Software Testing, Verification and Reliability 13(4) (2004)

6. Artho, C., Havelund, K., Biere, A.: Using Block-Local Atomicity to Detect StaleValue Concurrency Errors. In: 2nd International Symposium on Automated Technology for Verification and Analysis, Taiwan, October-November (2004)

7. ASML. http://research.microsoft.com/fse/asml

8. AspectJ. http://eclipse.org/aspectj

9. Barnett, M., Leino, K.R.M., Schulte, W.: The Spec\# Programming System: An Overview. In: Barthe, G., Burdy, L., Huisman, M., Lanet, J.-L., Muntean, T. (eds.) CASSIS 2004. LNCS, vol. 3362, Springer, Heidelberg (2005)

10. Barringer, H., Goldberg, A., Havelund, K., Sen, K.: Program Monitoring with LTL in Eagle. In: Parallel and Distributed Systems: Testing and Debugging (PADTAD 2004), Santa Fee, New Mexico, USA, April (2004)

11. Barringer, H., Goldberg, A., Havelund, K., Sen, K.: Rule-Based Runtime Verification. In: Steffen, B., Levi, G. (eds.) VMCAI 2004. LNCS, vol. 2937, Springer, Heidelberg (2004)

12. BCEL. http://jakarta.apache.org/bcel

13. Bensalem, S., Bozga, M., Krichen, M., Tripakis, S.: Testing Conformance of RealTime Applications by Automatic Generation of Observers. In: Proceedings of the 4th International Workshop on Runtime Verification (RV 2004) [1], pp. 19-38 (2004)

14. Bensalem, S., Fernandez, J.-C., Havelund, K., Mounier, L.: Confirmation of Deadlock Potentials Detected by Runtime Analysis. In: Parallel and Distributed Systems: Testing and Debugging (PADTAD 2006), Portland, Maine, USA (July 2006)

15. Bensalem, S., Havelund, K.: Scalable Dynamic Deadlock Analysis of MultiThreaded Programs. In: Parallel and Distributed Systems: Testing and Debugging (PADTAD - 3), IBM Verification Conference, Haifa, Israel, November 2005. LNCS (2005)

16. Chen, F., D'Amorim, M., Roşu, G.: Checking and Correcting Behaviors of Java Programs at Runtime with Java-MOP. In: Proceedings of the 5th International Workshop on Runtime Verification (RV 2005) [1] (2005)

17. Clavel, M., Durán, F.J., Eker, S., Lincoln, P., Martí-Oliet, N., Meseguer, J., Quesada, J.F.: The Maude System. In: Narendran, P., Rusinowitch, M. (eds.) RTA 1999. LNCS, vol. 1631, pp. 240-243. Springer, Heidelberg (1999)

18. Daikon. http://pag.csail.mit.edu/daikon

19. D'Amorim, M., Havelund, K.: Runtime Verification for Java. In: Workshop on Dynamic Program Analysis (WODA 2005) (March 2005)

20. D'Amorim, M., Rosu, G.: Efficient Monitoring of Omega-Languages. In: Etessami, K., Rajamani, S.K. (eds.) CAV 2005. LNCS, vol. 3576, Springer, Heidelberg (2005)

21. D'Angelo, B., Sankaranarayanan, S., Sanchez, C., Robinson, W., Finkbeiner, B., Sipma, H.B., Mehrotra, S., Manna, Z.: LOLA: Runtime Monitoring of Synchronous Systems. In: 12th International Symposium on Temporal Representation and Reasoning (TIME 2005), pp. 166-174 (2005)

22. Drusinsky, D.: The Temporal Rover and the ATG Rover. In: Havelund, K., Penix, J., Visser, W. (eds.) SPIN 2000. LNCS, vol. 1885, pp. 323-330. Springer, Heidelberg (2000) 
23. Drusinsky, D.: Monitoring Temporal Rules Combined with Time Series. In: Hunt Jr., W.A., Somenzi, F. (eds.) CAV 2003. LNCS, vol. 2725, pp. 114-118. Springer, Heidelberg (2003)

24. Drusinsky, D.: Semantics and Runtime Monitoring of TLCharts: Statechart Automata with Temporal Logic Conditioned Transitions. In: Proceedings of the 4th International Workshop on Runtime Verification (RV 2004) [1], pp. 2-18 (2004)

25. Eiffel. http://www.eiffel.com

26. Eytani, Y., Havelund, K., Stoller, S., Ur, S.: Toward a Framework and Benchmark for Testing Tools for Multi-Threaded Programs. In: Concurrency and Computation: Practice and Experience (to appear, 2005)

27. Finkbeiner, B., Sankaranarayanan, S., Sipma, H.: Collecting Statistics over Runtime Executions. In: Proceedings of the 2nd International Workshop on Runtime Verification (RV 2002) [1], pp. 36-55 (2002)

28. Finkbeiner, B., Sipma, H.: Checking Finite Traces using Alternating Automata. In: Proceedings of the 1st International Workshop on Runtime Verification (RV 2001)[1], pp. 44-60 (2001)

29. Flanagan, C., Freund, S.: Atomizer: A Dynamic Atomicity Checker for Multithreaded Programs. SIGPLAN Not. 39(1), 256-267 (2004)

30. Giannakopoulou, D., Havelund, K.: Automata-Based Verification of Temporal Properties on Running Programs. In: Proceedings, International Conference on Automated Software Engineering (ASE 2001), Coronado Island, California. ENTCS, pp. 412-416 (2001)

31. Goldberg, A., Havelund, K.: Instrumentation of Java Bytecode for Runtime Analysis. In: Fifth ECOOP Workshop on Formal Techniques for Java-like Programs (FTfJP 2003), Darmstadt, Germany (July 2003)

32. Harel, D.: Statecharts: A Visual Formalism For Complex Systems. Science of Computer Programming 8, 231-274 (1987)

33. Harrow, J.: Runtime Checking of Multithreaded Applications with Visual Threads. In: Havelund, K., Penix, J., Visser, W. (eds.) SPIN 2000. LNCS, vol. 1885, pp. 331342. Springer, Heidelberg (2000)

34. Havelund, K.: Using Runtime Analysis to Guide Model Checking of Java Programs. In: Havelund, K., Penix, J., Visser, W. (eds.) SPIN 2000. LNCS, vol. 1885, pp. 245 264. Springer, Heidelberg (2000)

35. Havelund, K., Roşu, G.: Monitoring Java Programs with Java PathExplorer. In: Proceedings of the 1st International Workshop on Runtime Verification (RV 2001)[1], pp. 97-114 (2001)

36. Havelund, K., Roşu, G.: Monitoring Programs using Rewriting. In: Proceedings, International Conference on Automated Software Engineering (ASE 2001), Coronado Island, California. Institute of Electrical and Electronics Engineers, pp. 135-143 (2001)

37. Havelund, K., Roşu, G.: An Overview of the Runtime Verification Tool Java PathExplorer. Formal Methods in System Design 24(2) (March 2004)

38. Havelund, K., Roşu, G.: Efficient Monitoring of Safety Properties. Software Tools for Technology Transfer 6(2), 158-173 (2004)

39. Havelund, K., Roşu, G.: Synthesizing Monitors for Safety Properties. In: Katoen, J.-P., Stevens, P. (eds.) ETAPS 2002 and TACAS 2002. LNCS, vol. 2280, pp. 342356. Springer, Heidelberg (2002), Best paper award

40. Jass. http://csd.informatik.uni-oldenburg.de/ jass

41. JML. http://www.cs.iastate.edu/ leavens/JML 
42. Kiczales, G., Hilsdale, E., Hugunin, J., Kersten, M., Palm, J., Griswold, W.G.: An Overview of AspectJ. In: Knudsen, J.L. (ed.) ECOOP 2001. LNCS, vol. 2072, pp. 327-353. Springer, Heidelberg (2001)

43. Kim, M., Kannan, S., Lee, I., Sokolsky, O.: Java-MaC: a Run-time Assurance Tool for Java. In: Proceedings of the 1st International Workshop on Runtime Verification (RV 2001)[1] (2001)

44. Larch. http://www.cs.iastate.edu/larch-faq-webboy.html

45. Patterns. http://patterns.projects.cis.ksu.edu

46. PSL/Sugar. http://www.pslsugar.org

47. PVS. http://pvs.csl.sri.com

48. RAISE. http://spd-web.terma.com/Projects/RAISE

49. Roşu, G., Havelund, K.: A Rewriting-based Approach to Trace Analysis. Automated Software Engineering 12(2), 151-197 (2005)

50. Savage, S., Burrows, M., Nelson, G., Sobalvarro, P., Anderson, T.: Eraser: A Dynamic Data Race Detector for Multithreaded Programs. ACM Transactions on Computer Systems 15(4), 391-411 (1997)

51. Sen, K., Roşu, G.: Generating Optimal Monitors for Extended Regular Expressions. In: Proceedings of the 3rd International Workshop on Runtime Verification (RV 2003)[1], pp. 162-181 (2003)

52. Sen, K., Roşu, G., Agha, G.: Detecting Errors in Multithreaded Programs by Generalized Predictive Analysis of Executions. In: Steffen, M., Zavattaro, G. (eds.) FMOODS 2005. LNCS, vol. 3535, Springer, Heidelberg (2005)

53. Specware. http://www.specware.org

54. SPIN. http://spinroot.com

55. Stolz, V., Bodden, E.: Temporal Assertions using AspectJ. In: Fifth Workshop on Runtime Verification (RV 2005). Electronic Notes in Theoretical Computer Science, Elsevier Science Publishers, Amsterdam (2005)

56. T-UPPAAL http://www.cs.aau.dk/ marius/tuppaal

57. Thati, P., Rosu, G.: Monitoring Algorithms for Metric Temporal Logic Specifications. In: Proceedings of the 4th International Workshop on Runtime Verification (RV 2004)[1], pp. 131-147 (2004)

58. Valgrind. http://valgrind.org

59. Vanderperren, W., Suvé, D., Augustina Cibrán, M., De Fraine, B.: Stateful Aspects in JAsCo. In: Workshop on Software Composition, ETAPS 2005 (2005)

60. VDM. http://www.csr.ncl.ac.uk/vdm

61. Visser, W., Havelund, K., Brat, G., Park, S.: Model Checking Programs. In: Proceedings of ASE 2000: The 15th IEEE International Conference on Automated Software Engineering, September 2000, IEEE CS Press, Los Alamitos (2000)

62. Walker, R.J., Viggers, K.: Implementing Protocols via Declarative Event Patterns. In: Taylor, R.N., Dwyer, M.B. (eds.) 12th International Symposium on the Foundations of Software Engineering, ACM, New York (2004)

63. Wang, L., Stoller, S.: Run-Time Analysis for Atomicity. In: Proceedings of the 3rd International Workshop on Runtime Verification (RV 2003) [1] (2003) 